\title{
Factors affecting healing time of postoperative pancreatic fistula in patients undergoing pancreaticoduodenectomy
}

\author{
YOSHITO TOMIMARU, KOZO NOGUCHI, SHINGO NOURA, HIROSHI IMAMURA, \\ TAKASHI IWAZAWA and KEIZO DONO \\ Department of Surgery, Toyonaka Municipal Hospital, Toyonaka, Osaka 560-8565, Japan
}

Received November 19, 2018; Accepted February 20, 2019

DOI: $10.3892 / \mathrm{mco} .2019 .1812$

\begin{abstract}
Clinically-relevant postoperative pancreatic fistula (CR-POPF) is one of the most serious complications following pancreaticoduodenectomy. While many investigators have studied risk factors for the development of CR-POPF, few studies have focused on time needed for CR-POPF healing in patients who develop this complication. The present study enrolled 38 cases in whom CR-POPF developed following pancreaticoduodenectomy $(24.1 \%)$ out of 158 cases receiving treatment with pancreaticoduodenectomy between 2009 and 2017 for periampullary diseases. CR-POPF healing time, was defined as the length of time from the day of pancreaticoduodenectomy to the day when intraabdominal drainage tubes used for CR-POPF treatment were removed, were assessed and factors affecting the healing time were investigated. The mean CR-POPF healing time was 40.2 21.7 days (median; 35 days, range; 10-110 days). Univariate analysis demonstrated a significant relationship between CP-POPE healing time and sex, intraoperative blood loss, and type of pancreaticojejunostomy procedure. Multivariate Cox regression analysis using these factors revealed that intraoperative blood loss and type of pancreaticojejunostomy procedure were significant independent factors for the length of CR-POPF healing time. CR-POPF healing time following pancreaticoduodenectomy was $40.2 \pm 21.7$ days on average (median, 35 days; range 10-110 days). Intraoperative blood loss and type of pancreaticojejunostomy procedure were identified as significant independent factors associated with the healing time. These results will help with earlier recovery from CR-POPF.
\end{abstract}

Correspondence to: Dr Yoshito Tomimaru, Department of Surgery, Toyonaka Municipal Hospital, 4-14-1 Shibahara, Toyonaka, Osaka 560-8565, Japan

E-mail: ytomimaru@gmail.com

Key words: pancreatic fistula, intraoperative blood loss, modified Blumgart anastomosis

\section{Introduction}

Postoperative pancreatic fistula (POPF) is generally recognized as one of the most common complications following pancreaticoduodenectomy (PD) (1-3). Although perioperative management and operative techniques used during pancreatic surgery have improved, it occurs in approximately 5 to $30 \%$ of all PD procedures $(2,4-6)$. Clinically-relevant POPF (CR-POPF) can trigger subsequent postoperative complications such as intraabdominal abscess and pseudoaneurysm associated with postpancreatectomy hemorrhage, resulting in longer hospital stays, increased treatment costs, and even postoperative mortality. Therefore, identifying patients at high risk for developing CR-POPF is considered important for improving the clinical outcome in patients undergoing PD. In this regard, many studies have identified predictive factors for developing CR-POPF such as body mass index, diameter of the main pancreatic duct (MPD), and pancreatic consistency (7-11). In addition to such predictors of CR-POPF development, treatment of POPF after it develops is also important for improving clinical outcomes. When considering the treatment for CR-POPF, while the therapeutic options themselves are actually important, healing time is the most important issue regardless of the choice of the therapeutic options. However, few studies have focused on the time needed for CR-POPF healing, though many investigators have studied risk factors for developing this complication. Therefore, in the present study, our aim was to assess the time needed for CR-POPF healing after PD and, furthermore, to investigate factors affecting the healing time based on our experiences.

\section{Patients and methods}

A total of consecutive 158 cases underwent PD between 2009 and 2017 for periampullary diseases at the Department of Surgery, Toyonaka Municipal Hospital. This study excluded cases where curative resection was not achieved and those which required resection of other organs in addition to PD. Among the 158 cases, CR-POPF developed after PD in 38 cases (24.1\%), and these patients were enrolled in the present study. The clinical and surgical characteristics of these patients are summarized in Table I. The time needed for CR-POPF healing was assessed, and factors affecting the healing time were investigated. 
Subtotal stomach-preserving PD was basically adopted as the PD procedure in the cases. With regard to reconstruction after the resection, a modified Child method, with pancreaticojejunostomy (PJ), choledochoduodenostomy, and gastrojejunostomy with Braun's anastomosis, were performed. The Kakita method or a modified Blumgart anastomosis method was applied as techniques for pancreaticojejunostomy based on the surgeon's preference $(12,13)$. Briefly, in patients with the Kakita method, in addition to duct-to-mucosa PJ anastomosis, the pancreatic parenchyma of the stump was approximated to jejunal seromuscular layer with nonabsorbable interrupted penetrating sutures. In patients with the modified Blumgart anastomosis group, a double-armed nonabsorbable suture was used to place a U-suture with both arms through the pancreatic stump and a longitudinal suture through the jejunal seromuscular layer. After the duct-to-mucosa PJ anastomosis, the outer anterior horizontal mattress sutures on the jejunum with the U-sutures were completed and tied on the anterior surface of the pancreas to cover the duct-to-mucosa anastomosis by jejunal serosa. Before completing the surgery, we placed 2 and 1 closed intraabdominal drainage tubes at the vicinity of pancreaticojejunostomy and hepaticojejunostomy, respectively. POPF was diagnosed and stratified by severity into biochemical leakage (BL), grade B, or grade C according to the International Study Group of Pancreatic Fistula definition (14). CR-POPF was defined as grade B or grade C POPF. Our treatment for CR-POPF was performed uniformly in all the patients. Briefly, for the treatment, the abdominal drainage was continued until disappearance of the abdominal cavity was confirmed at imaging modalities. Cure was judged to be when the intraabdominal drainage tubes used for the CR-POPF treatment were removed. The drainage tubes were changed every 1-2 weeks until their removal. During the treatment, oral intake was continued. No patients received somatostatin analogs. In this study, CR-POPF healing time was defined as the length of time (in days) from the day of the surgery to the day when CR-POPF was cured. The diameter of the MPD was measured at the resection line of the pancreas on enhanced CT images. Pancreatic consistency was judged as soft or hard based on intraoperative findings of the pancreas.

On the basis of extensive dialogue with the Human Ethics Review Committee of Toyonaka Municipal Hospital, approval for an opt-out consent method was given. The study received ethical approval for the use of an opt-out methodology, and the participation of the patients was obtained through the opt-out methodology (certificate no. 2018-05-07).

Data were expressed as mean \pm standard deviation. Differences between groups were assessed by Chi-square test, Fisher's exact test, or Mann-Whitney U test. Analysis of the healing time was performed based on cumulative healing rate calculated with the Kaplan-Meier method. Cumulative healing rates in subgroups were compared with the log-rank test in univariate analysis. Multivariate analysis was performed using Cox proportional hazard regression for identifying independent variables associated with healing time. A median was used for turning a continuous variable into a categorical one. Statistical analyses were performed using StatView (version 5.0; SAS Institute Inc., Cary, NC, USA). A P $<0.05$ was considered significant.

\section{Results}

Among the 158 patients who underwent PD, CR-POPF developed in 38 cases $(24.1 \%)$, while CR-POPF was not identified postoperatively in the remaining 120 cases (75.9\%). Among the 38 cases with CR-POPF, there were 35 cases with grade $\mathrm{B}$ and 3 cases with grade $\mathrm{C}$. Outcome of the patients with grade $\mathrm{C}$ was death derived from POPF. The remaining 120 cases without CR-POPF included 35 cases with BL and 85 cases without BL or CR-POPF. The clinical and surgical characteristics of the cases with and without CR-POPF are shown in Table I. Cases with and without CR-POPF were significantly different in age, weight, body mass index, disease, diameter of MPD, and pancreatic consistency, which is consistent with previous reports. CR-POPF was treated during hospitalization in all patients, with a mean duration of $40.2 \pm 21.7$ days (median, 35 days; range 10-110 days). The time needed for healing of CR-POPF in individual cases is shown in Fig. 1. Univariate analysis using various factors associated with CR-POPF healing time is summarized in Table II. The univariate analysis showed a significant relationship between sex (male vs. female), procedure for pancreaticojejunostomy (Kakita vs. modified Blumgart anastomosis), and intraoperative blood loss ( $<696$ vs. $\geq 696 \mathrm{ml})(\mathrm{P}=0.0390, \mathrm{P}=0.0262$ and $\mathrm{P}=0.0204$, respectively). Cumulative healing rates after the surgery calculated with the Kaplan-Meier method and stratified by these significant factors are shown in Fig. 2A-C. The remaining factors including age, height, weight, body mass index, preoperative laboratory parameters such as hemoglobin, lymphocytes, prothrombin time, total protein, albumin, total cholesterol, and cholinesterase level, prognostic nutritional index defined by Onodera et al (15), disease, diameter of MPD, presence of preoperative biliary drainage, pancreatic consistency, pancreaticojejunostomy procedure, presence of combined resection of the portal vein, operation time, presence of homologous blood transfusion, and presence of postoperative complication other than CR-POPF were not significantly associated with CR-POPF healing time.

Next, to identify significant independent factors for the time needed for CR-POPF healing, multivariate Cox regression analysis was performed using factors identified as significant variables in the univariate analysis (Table II). The multivariate analysis demonstrated that intraoperative blood loss ( $<696$ vs. $\geq 696 \mathrm{ml}$ ) and procedure for pancreaticojejunostomy (Kakita vs. modified Blumgart anastomosis) were significant independent factors for CR-POPF healing time ( $\mathrm{P}=0.0305$ and $\mathrm{P}=0.0429$, respectively) (Table II). Using these independent factors, we proposed a practical stratification of the CR-POPF patients for the prediction of the healing time of CR-POPF. The stratification was as follows; Group I included patients with smaller intraoperative blood loss $(<696 \mathrm{ml})$ and adoption of modified Blumgart anastomosis for the reconstruction, Group II included those with larger intraoperative blood loss $(/ \geq 696 \mathrm{ml})$ and adoption of modified Blumgart anastomosis and those with smaller intraoperative blood loss $(<696 \mathrm{ml})$ and adoption of Kakita method, and Group III included those with larger intraoperative blood loss $(/ \geq 696 \mathrm{ml})$ and adoption of Kakita method. When stratified by the groups, cumulative healing rates after the surgery were significantly different among the groups ( $\mathrm{P}=0.0020)$ (Fig. 2D). Median healing time 
Table I. Comparison of the perioperative factors of patients with and without CR-POPF.

\begin{tabular}{|c|c|c|c|c|}
\hline Factor & $\begin{array}{l}\text { All cases } \\
(n=158)\end{array}$ & $\begin{array}{c}\text { CR-POPF }(-) \\
\quad(n=120)\end{array}$ & $\begin{array}{c}\text { CR-POPF }(+) \\
\quad(n=38)\end{array}$ & P-value \\
\hline \multicolumn{5}{|l|}{ Preoperative factors } \\
\hline Age (years) & $70 \pm 9$ & $70 \pm 10$ & $70 \pm 7$ & 0.8397 \\
\hline Sex (male/female) & $93 / 65$ & $65 / 55$ & $28 / 10$ & 0.0331 \\
\hline Height (cm) & $159.6 \pm 9.6$ & $159.2 \pm 9.7$ & $160.6 \pm 9.5$ & 0.4277 \\
\hline Weight (kg) & $55.0 \pm 9.8$ & $54.0 \pm 9.6$ & $58.1 \pm 9.9$ & 0.0223 \\
\hline Body mass index $\left(\mathrm{kg} / \mathrm{m}^{2}\right)$ & $21.5 \pm 2.8$ & $21.2 \pm 2.8$ & $22.4 \pm 2.6$ & 0.0205 \\
\hline Hemoglobin (g/dl) & $12.2 \pm 1.5$ & $12.1 \pm 1.4$ & $12.4 \pm 1.5$ & 0.2708 \\
\hline Lymphocyte $(/ \mu 1)$ & $1535 \pm 736$ & $1507 \pm 764$ & $1623 \pm 642$ & 0.3958 \\
\hline $\mathrm{PT}(\%)$ & $93 \pm 15$ & $93 \pm 14$ & $94 \pm 16$ & 0.7402 \\
\hline Total protein $(\mathrm{g} / \mathrm{dl})$ & $6.8 \pm 0.7$ & $6.8 \pm 0.7$ & $6.8 \pm 0.5$ & 0.3081 \\
\hline Albumin (g/dl) & $3.5 \pm 0.5$ & $3.6 \pm 0.5$ & $3.6 \pm 0.4$ & 0.4271 \\
\hline Total cholesterol (mg/dl) & $190 \pm 45$ & $188 \pm 46$ & $196 \pm 44$ & 0.4019 \\
\hline Cholinesterase (U/l) & $257 \pm 84$ & $251 \pm 87$ & $273 \pm 69$ & 0.1669 \\
\hline Amylase (U/l) & $107 \pm 81$ & $102 \pm 89$ & $98 \pm 39$ & 0.5639 \\
\hline Prognostic nutritional index & $43.0 \pm 6.6$ & $42.8 \pm 6.7$ & $44.0 \pm 5.7$ & 0.2360 \\
\hline Disease & & & & 0.0009 \\
\hline Pancreatic cancer & 55 & 52 & 3 & \\
\hline Bile duct cancer & 43 & 30 & 13 & \\
\hline Ampullary cancer & 17 & 11 & 6 & \\
\hline Others & 43 & 27 & 16 & \\
\hline Diameter of MPD (mm) & $4.1 \pm 1.9$ & $4.5 \pm 2.0$ & $2.8 \pm 1.1$ & $<0.0001$ \\
\hline Biliary drainage & & & & 0.3186 \\
\hline None & 77 & 60 & 17 & \\
\hline External & 16 & 14 & 2 & \\
\hline Internal & 65 & 46 & 19 & \\
\hline \multicolumn{5}{|l|}{ Intraoperative factors } \\
\hline Pancreatic consistency (soft/hard) & $89 / 69$ & $52 / 68$ & $37 / 1$ & $<0.0001$ \\
\hline Pancreaticojejunostomy procedure (Kakita/Blumgart) & $85 / 73$ & $60 / 60$ & $25 / 13$ & 0.0889 \\
\hline Combined resection of portal vein $(-/+)$ & $118 / 40$ & $86 / 34$ & $32 / 6$ & $>0.9999$ \\
\hline Operation time (min) & $428 \pm 99$ & $425 \pm 100$ & $439 \pm 98$ & 0.4618 \\
\hline Intraoperative blood loss (mL) & $844 \pm 566$ & $814 \pm 538$ & $936 \pm 644$ & 0.2489 \\
\hline Homologous blood transfusion (-/+) & $132 / 26$ & $102 / 18$ & $30 / 8$ & 0.3805 \\
\hline \multicolumn{5}{|l|}{ Postoperative factors } \\
\hline Postoperative complication other than CR-POPF (-/+) & & $101 / 19$ & $28 / 10$ & 0.1457 \\
\hline
\end{tabular}

CR-POPF, clinically relevant postoperative pancreatic fistula; MPD, main pancreatic duct; PT, prothrombin time.

of CR-POPF was 24 days in Group I, 36 days in Group II, and 45 days in Group III.

\section{Discussion}

The present study focused on the time needed for CR-POPF healing in patients who had developed this complication after PD. We clarified the actual healing time and successfully identified two statistically significant independent factors associated with the time needed for healing of CR-POPF. Based on the fact that CR-POPF potentially causes subsequent critical postoperative complications such as intraabdominal abscess and pseudoaneurysm associated with postpancreatectomy hemorrhage, both predicting and treating CR-POPF are clinically significant issues. With regard to its prediction, owing to previous studies some factors have been identified as being predictive for CR-POPF development such as body mass index, indication disease for the surgical treatment, diameter of the main pancreatic duct, texture of the pancreatic parenchyma, and amount of intraoperative bleeding $(7-11,16,17)$. On the other hand, few studies have focused on treatment of CR-POPF. Especially, to the best of our knowledge, the time needed for CR-POPF healing has not yet been discussed. Based on the above-mentioned nature of CR-POPF, and considering that CR-POPF develops in some patients regardless of the predictive factors, investigation into the time needed for 


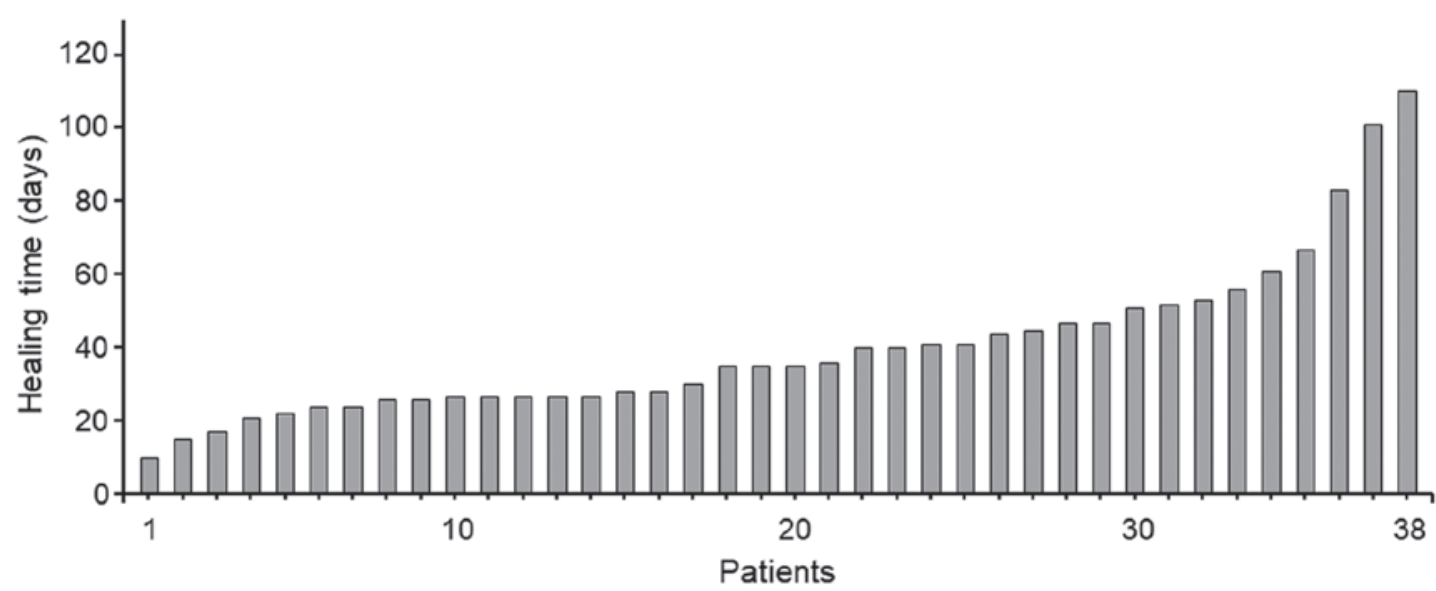

Figure 1. Time needed for CR-POPF healing in individual cases. The cases (\#1-38) were arranged by the length of the healing time. CR-POPF, clinically relevant postoperative pancreatic fistula.
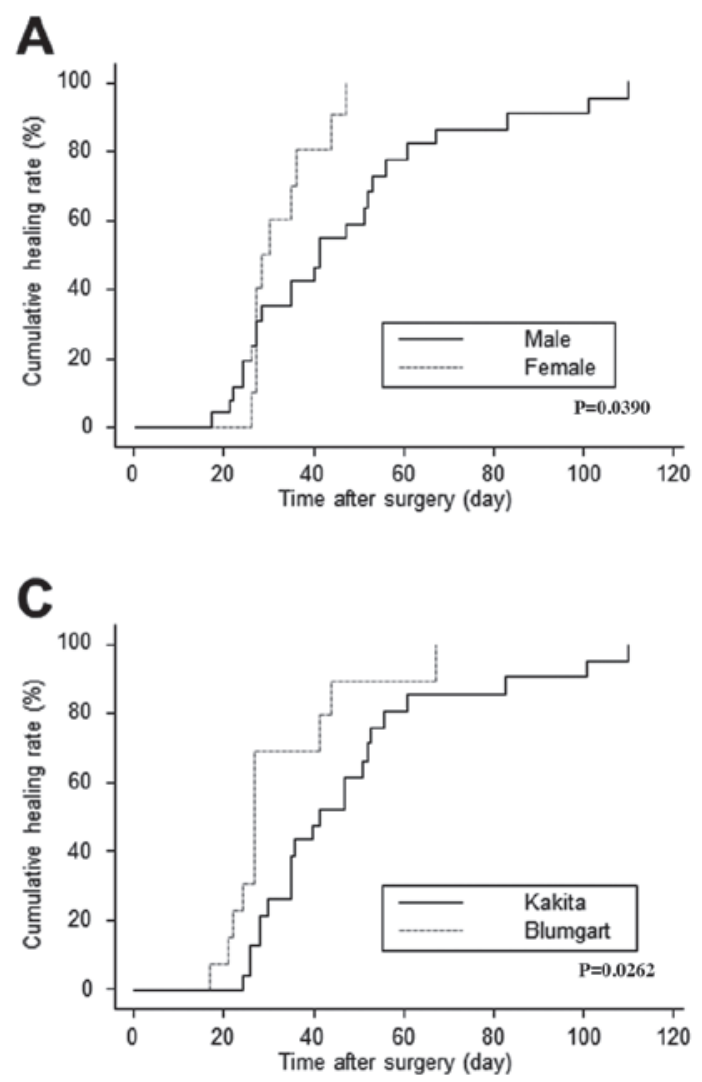

B

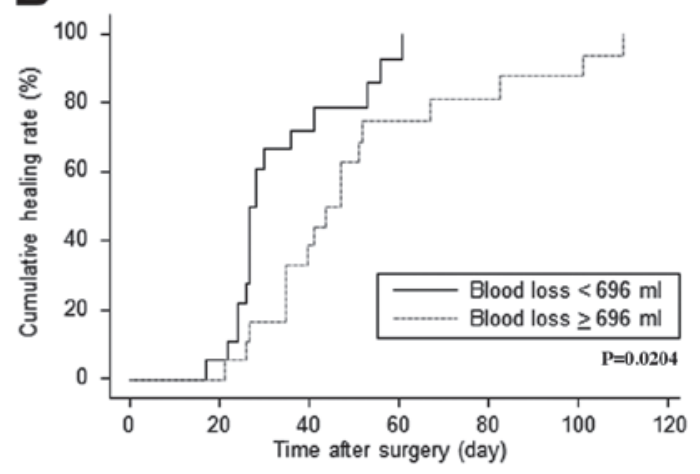

D

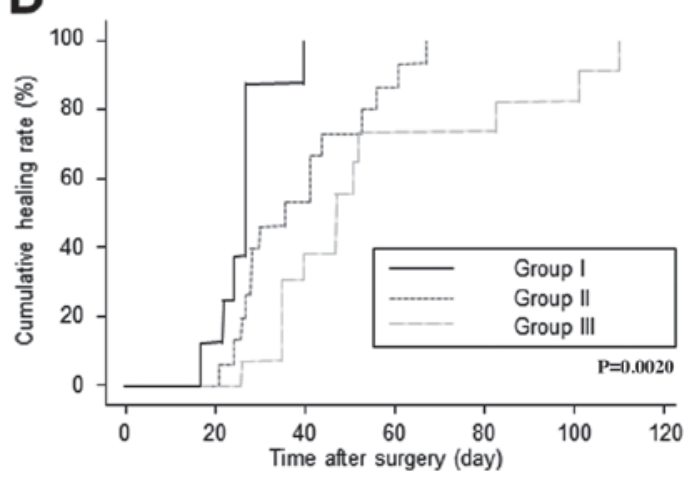

Figure 2. Cumulative CR-POPF healing rate following surgery. The rates were calculated with the Kaplan-Meier method and described graphically with stratification by significant factors; (A) sex, (B) intraoperative blood loss, and (C) procedure used for pancreaticojejunostomy. (D) The rates calculated with the Kaplan-Meier method were described with liners with a proposed stratification; Group I included patients with smaller intraoperative blood loss ( $<696 \mathrm{ml})$ and use of modified Blumgart anastomosis for the reconstruction, Group II included those with larger intraoperative blood loss ( $/ \geq 696 \mathrm{ml})$ and use of modified Blumgart anastomosis and those with smaller intraoperative blood loss $(<696 \mathrm{ml})$ and use of Kakita method, and Group III included those with larger intraoperative blood loss ( $/ \geq 696 \mathrm{ml})$ and use of Kakita method. CR-POPF, clinically relevant postoperative pancreatic fistula.

healing should be discussed more frequently. Earlier recovery after CR-POPF develops could potentially lead to a lower incidence of the subsequent critical problems related to CR-POPF as mentioned above. This background led us to feel that the present study was worth reporting.

The two factors identified as significant independent factors associated with CR-POPF healing time in the present study were intraoperative blood loss and the procedure used for pancreaticojejunostomy. While these factors were identified as being associated with healing time for the first time, some studies have reported a significant relationship between these factors and CR-POPF development. For example, with regard to intraoperative blood loss, Kawai et al reported that intraoperative bleeding $>1,000 \mathrm{ml}$ is a significant predictive factor for POPF based on a study by the Japanese Society of Hepato-Biliary-Pancreatic Surgery (16). Yang et al also 
Table II. Determinants of CR-POPF healing time in patients with CR-POPF.

\begin{tabular}{|c|c|c|c|c|c|}
\hline \multirow[b]{2}{*}{ Preoperative factors } & \multirow[b]{2}{*}{ No. of patients } & \multirow{2}{*}{$\frac{\text { Univariate }}{\text { P-value }}$} & \multicolumn{3}{|c|}{ Multivariate } \\
\hline & & & OR & $95 \% \mathrm{CI}$ & P-value \\
\hline Age (year) $(<65$ vs. $\geq 65)$ & $19 / 19$ & 0.9280 & & & \\
\hline Sex (male vs. female) & $28 / 10$ & 0.0390 & 1.873 & $0.799-4.388$ & 0.1487 \\
\hline Height $(\mathrm{cm})(<162$ vs. $\geq 162)$ & $19 / 19$ & 0.8332 & & & \\
\hline Weight (kg) (<57 vs. $\geq 57)$ & $19 / 19$ & 0.5485 & & & \\
\hline Body mass index $\left(\mathrm{kg} / \mathrm{m}^{2}\right)(<22 \mathrm{vs} . \geq 22)$ & $19 / 19$ & 0.9604 & & & \\
\hline Hemoglobin (g/dl) (<12.9 vs. $\geq 12.9)$ & $19 / 19$ & 0.3433 & & & \\
\hline Lymphocyte $(/ \mu 1)(<1525$ vs. $\geq 1525)$ & $19 / 19$ & 0.8480 & & & \\
\hline PT $(\%)(<93$ vs. $\geq 93)$ & $19 / 19$ & 0.2522 & & & \\
\hline Total protein $(\mathrm{g} / \mathrm{dl})(<6.9 \mathrm{vs} . \geq 6.9)$ & $19 / 19$ & 0.3166 & & & \\
\hline Albumin (g/dl) & $19 / 19$ & 0.3185 & & & \\
\hline Total cholesterol (mg/dl) (<190 vs. $\geq 190)$ & $19 / 19$ & 0.4927 & & & \\
\hline Cholinesterase (U/1) (<273 vs. $\geq 273)$ & $19 / 19$ & 0.3956 & & & \\
\hline Amylase (U/l) (<85 vs. $\geq 85)$ & $19 / 19$ & 0.2151 & & & \\
\hline Prognostic nutritional index (<46 vs. $\geq 46)$ & $19 / 19$ & 0.7374 & & & \\
\hline Disease (pancreatic cancer vs. others) & $3 / 35$ & 0.5887 & & & \\
\hline Diameter of MPD (mm) (<2.8 vs. $\geq 2.8)$ & $19 / 19$ & 0.5152 & & & \\
\hline Biliary drainage (- vs. +) & $17 / 21$ & 0.9065 & & & \\
\hline \multicolumn{6}{|l|}{ Intraoperative factors } \\
\hline Pancreatic consistency (Soft/Hard) & $37 / 1$ & 0.7975 & & & \\
\hline Pancreaticojejunostomy procedure (Kakita/Blumgart) & $25 / 13$ & 0.0262 & 2.225 & $1.026-4.825$ & 0.0429 \\
\hline Combined resection of portal vein (- vs. +) & $32 / 6$ & 0.2095 & & & \\
\hline Operation time (min) (<430 vs. $\geq 430)$ & $19 / 19$ & 0.4896 & & & \\
\hline Intraoperative blood loss (ml) (<696 vs. $\geq 696)$ & $19 / 19$ & 0.0204 & 0.429 & $0.200-0.923$ & 0.0305 \\
\hline Homologous blood transfusion (- vs. +) & $30 / 8$ & 0.9297 & & & \\
\hline \multicolumn{6}{|l|}{ Postoperative factors } \\
\hline Postoperative complication other than CR-POPF (-/+) & $28 / 10$ & 0.5091 & & & \\
\hline
\end{tabular}

OR, odds ratio, 95\% CI, 95\% confidence interval; CR-POPF, clinically relevant postoperative pancreatic fistula; MPD, main pancreatic duct; PT, prothrombin time.

reported a similar study (17). As for the procedure used for pancreaticojejunostomy, Fujii et al reported that the rate of CR-POPF formation was significantly lower in patients where the modified Blumgart anastomosis was used than in patients where the Kakita method was used (12). Thus, it can be considered that these two factors are significantly associated not only with POPF development, but also with CR-POPF healing time.

As for the clinical application of the results of this study, it is important to consider the merits of a shorter healing time for CR-POPF. Shortening the time needed for CR-POPF healing has some potential clinical advantages of which one is lowering the incidence of subsequent critical postoperative complications due to CR-POPF. The second is decreasing the cost of hospitalization due to the shortened hospitalized period. Finally, shorter healing allows for smoother administration of adjuvant therapy when the patients have cancers for which adjuvant therapy is proven to be oncologically effective $(18,19)$. In this regard, keeping the effect of intraoperative blood loss and the procedure used for pancreaticojejunostomy and the effect of these factors on CR-POPF healing time in mind could potentially ensure these advantages.
The present study has several limitations. The most obvious one is the study design, which was retrospective and it was not a prospective, randomized, controlled study. Therefore, there could be certain biases in several points. For example, a bias exists in the selection of the procedure for pancreaticojejunostomy. As mentioned above, the Kakita method or modified Blumgart anastomosis methods were applied based on the surgeon's preference, but the latter is more recently developed than the former, which suggest that patients' backgrounds in the cases where each method was used are potentially different even though there were no statistically significant differences in other background factors between the two groups. The second limitation is that the data for the present study were based on the experience of a single institution. This may produce some bias in the preoperative management of the patients such as surgical procedures, method for measuring intraoperative blood loss, or management of the drainage tubes. Taken together, the study has several potential limitations and, furthermore, the results of the study are not validated. In future, these results should be validated in a multi-institutional, prospective, randomized, controlled trial, using certain criteria as mentioned above. 
In summary, we examined the time needed for CR-POPF healing after PD and identified two statistically significant independent factors associated with the healing time; intraoperative blood loss and the type of procedure used for pancreaticojejunostomy. The findings could provide information that leads to earlier recovery from CR-POPF.

\section{Acknowledgements}

Not applicable.

\section{Funding}

No funding was relieved.

\section{Availability of data and materials}

All data generated or analyzed during this study are included in this published article.

\section{Authors' contributions}

YT was responsible for the concept and design of the study and for data interpretation. $\mathrm{KN}$ assisted with data interpretation and production of the article. SN, HI and TI assisted in data interpretation and article preparation. KD supervised the project.

\section{Ethics approval and consent to participate}

Ethical approval for this study was obtained from the Human Ethics Review Committee of Toyonaka Municipal Hospital (Certificate no. 2018-05-07). Written informed consent was obtained from the patients.

\section{Patient consent for publication}

Not applicable.

\section{Conflicts of interest}

The authors declare that they have no competing interests.

\section{References}

1. de Castro SM, Busch OR, van Gulik TM, Obertop H and Gouma DJ: Incidence and management of pancreatic leakage after pancreatoduodenectomy. Br J Surg 92: 1117-1123, 2005.

2. Kirihara Y, Takahashi N, Hashimoto Y, Sclabas GM, Khan S, Moriya T, Sakagami J, Huebner M, Sarr MG and Farnell MB: Prediction of pancreatic anastomotic failure after pancreatoduodenectomy: The use of preoperative, quantitative computed tomography to measure remnant pancreatic volume and body composition. Ann Surg 257: 512-519, 2013.

3. Fu SJ, Shen SL, Li SQ, Hu WJ, Hua YP, Kuang M, Liang LJ and Peng BG: Risk factors and outcomes of postoperative pancreatic fistula after pancreatico-duodenectomy: An audit of 532 consecutive cases. BMC Surg 15: 34, 2015.

4. Hashimoto Y and Traverso LW: Pancreatic anastomotic failure rate after pancreaticoduodenectomy decreases with microsurgery. J Am Coll Surg 211: 510-521, 2010.

5. Pecorelli N, Balzano G, Capretti G, Zerbi A, Di Carlo V and Braga M: Effect of surgeon volume on outcome following pancreaticoduodenectomy in a high-volume hospital. J Gastrointest Surg 16: 518-523, 2012.
6. Poon RT, Fan ST, Lo CM, Ng KK, Yuen WK, Yeung C and Wong J: External drainage of pancreatic duct with a stent to reduce leakage rate of pancreaticojejunostomy after pancreaticoduodenectomy: A prospective randomized trial. Ann Surg 246: 425-435, 2007.

7. Aoki S, Miyata H, Konno H, Gotoh M, Motoi F, Kumamaru H, Wakabayashi G, Kakeji Y, Mori M, Seto Y, et al: Risk factors of serious postoperative complications after pancreaticoduodenectomy and risk calculators for predicting postoperative complications: A nationwide study of 17,564 patients in Japan. J Hepatobiliary Pancreat Sci 24: 243-251, 2017.

8. Gaujoux S, Cortes A, Couvelard A, Noullet S, Clavel L, Rebours V, Lévy P, Sauvanet A, Ruszniewski P and Belghiti J: Fatty pancreas and increased body mass index are risk factors of pancreatic fistula after pancreaticoduodenectomy. Surgery 148: 15-23, 2010.

9. Hu BY, Wan T, Zhang WZ and Dong JH: Risk factors for postoperative pancreatic fistula: Analysis of 539 successive cases of pancreaticoduodenectomy. World J Gastroenterol 22: 7797-7805, 2016.

10. Shubert CR, Wagie AE, Farnell MB, Nagorney DM, Que FG, Reid Lombardo KM, Truty MJ, Smoot RL and Kendrick ML: Clinical Risk Score to Predict Pancreatic Fistula after Pancreatoduodenectomy: Independent External Validation for Open and Laparoscopic Approaches. J Am Coll Surg 221: 689-698, 2015.

11. Tranchart H, Gaujoux S, Rebours V, Vullierme MP, Dokmak S, Levy P, Couvelard A, Belghiti J and Sauvanet A: Preoperative CT scan helps to predict the occurrence of severe pancreatic fistula after pancreaticoduodenectomy. Ann Surg 256: 139-145, 2012.

12. Fujii T, Sugimoto H, Yamada S, Kanda M, Suenaga M, Takami H, Hattori M, Inokawa Y, Nomoto S, Fujiwara M, et al: Modified Blumgart anastomosis for pancreaticojejunostomy: Technical improvement in matched historical control study. J Gastrointest Surg 18: 1108-1115, 2014.

13. Kakita A, Yoshida M and Takahashi T: History of pancreaticojejunostomy in pancreaticoduodenectomy: Development of a more reliable anastomosis technique. J Hepatobiliary Pancreat Surg 8: 230-237, 2001.

14. Bassi C, Marchegiani G, Dervenis C, Sarr M, Abu Hilal M, AdhamM, Allen P, Andersson R, Asbun HJ, Besselink MG, et al; International Study Group on Pancreatic Surgery (ISGPS): The 2016 update of the International Study Group (ISGPS) definition and grading of postoperative pancreatic fistula: 11 Years After. Surgery 161: 584-591, 2017.

15. Onodera T, Goseki N and Kosaki G: Prognostic nutritional index in gastrointestinal surgery of malnourished cancer patients. Nihon Geka Gakkai Zasshi 85: 1001-1005, 1984 (In Japanese).

16. Kawai M, Kondo S, Yamaue H, Wada K, Sano K, Motoi F, Unno M, Satoi S, Kwon AH, Hatori T, et al: Predictive risk factors for clinically relevant pancreatic fistula analyzed in 1,239 patients with pancreaticoduodenectomy: Multicenter data collection as a project study of pancreatic surgery by the Japanese Society of Hepato-Biliary-Pancreatic Surgery. J Hepatobiliary Pancreat Sci 18: 601-608, 2011.

17. Yang H, Lu XF, Xu YF, Liu HD, Guo S, Liu Y and Chen YX: Application of air insufflation to prevent clinical pancreatic fistula after pancreaticoduodenectomy. World J Gastroenterol 21: 1872-1879, 2015.

18. Uesaka K, Boku N, Fukutomi A, Okamura Y, Konishi M, Matsumoto I, Kaneoka Y, Shimizu Y, Nakamori S, Sakamoto H, et al; JASPAC 01 Study Group: Adjuvant chemotherapy of S-1 versus gemcitabine for resected pancreatic cancer: A phase 3 , open-label, randomised, non-inferiority trial (JASPAC 01). Lancet 388: 248-257, 2016.

19. Neoptolemos JP, Palmer DH, Ghaneh P, Psarelli EE, Valle JW, HalloranCM, Faluyi O, O'Reilly DA, Cunningham D, Wadsley J, et al; European Study Group for Pancreatic Cancer: Comparison of adjuvant gemcitabine and capecitabine with gemcitabine monotherapy in patients with resected pancreatic cancer (ESPAC-4): A multicentre, open-label, randomised, phase 3 trial. Lancet 389: 1011-1024, 2017. 